\title{
Tripolifosfato de sódio e hexametafosfato de sódio na prevenção do cálculo dentário em cães
}

[Sodium tripolyphosphate and sodium hexametaphosphate in preventing dental calculus accumulation in dogs]

\author{
A.B.F. Pinto ${ }^{1}$, F.M.O.B. Saad $^{2}$, C.A.L. Leite $^{3}$, A.A. Aquino ${ }^{1}$, M.P. Alves ${ }^{1}$, D.A.R. Pereira ${ }^{4}$ \\ ${ }^{1}$ Aluna de pós-graduação - UFLA - Lavras, MG \\ ${ }^{2}$ Departamento de Zootecnia - UFLA - Lavras, MG \\ ${ }^{3}$ Departamento de Medicina Veterinária - UFLA - Lavras, MG \\ ${ }^{4}$ Total Alimentos S.A. - Três Corações, MG
}

\begin{abstract}
RESUMO
Avaliou-se o efeito de dois fosfatos, tripolifosfato de sódio (TPF) e hexametafosfato de sódio (HMF), incorporados à ração seca sob diferentes formas, como cobertura do grânulo da ração e no interior da massa da ração, na prevenção do desenvolvimento de cálculo dentário em 25 cães. O delineamento experimental utilizado foi inteiramente ao acaso, com cinco tratamentos e cinco repetições. Os tratamentos experimentais foram: 1) ração seca; 2) ração seca com TPF incorporado na cobertura do grânulo da ração; 3) ração seca com TPF incorporado na massa da ração; 4) ração seca com HMF incorporado na cobertura do grânulo da ração; 5) ração seca com HMF incorporado na massa da ração. Os animais receberam as dietas por um período experimental de 90 dias e, após esse período, foram submetidos à avaliação da área de cálculo dentário formado. A inclusão do HMF na ração seca, tanto na cobertura dos grânulos como no interior da massa, e do TPF, como cobertura dos grânulos, reduziu o acúmulo de cálculo dentário em cães, comparada à dieta sem adição de fosfatos. O HMF foi o fosfato mais eficiente, ao reduzir o acúmulo de cálculo dentário em até $47 \%$. As formas de inclusão do HMF na ração não influenciaram o acúmulo de cálculo dentário. Houve redução do desenvolvimento de cálculo dentário pela incorporação do TPF como cobertura do grânulo da ração, comparada à inclusão deste fosfato no interior da massa da ração. Conclui-se que os fosfatos incorporados na ração seca reduzem o acúmulo de cálculo dentário em cães.
\end{abstract}

Palavras-chave: cão, cálculo dentário, tripolifosfato de sódio, hexametafosfato de sódio

\begin{abstract}
The effect of dry food treated with sodium tripolyphosphate (TPP) or sodium hexametaphosphate (HMP), as kibble coated or added into the interior of the kibble on the accumulation of dental calculus in 25 dogs was evaluated. The study used a completely randomized design with five treatments and five repetitions. The experimental treatments were: 1) dry food; 2) dry food coated with TPP; 3) dry food with TPP incorporated into the interior of the kibble; 4) dry food coated with HMP; 5) dry food with HMP incorporated into the interior of the kibble. The animals received the diets for a 90-day experimental period, and then, the teeth were clinically examined for the presence of calculus. The inclusion of HMP in the dry food, as kibble coated or added into the interior of the kibble, and the inclusion of TPP as kibble coated reduced the accumulation of dental calculus compared to the control group without anti-calculus agents. HMP was the most efficient phosphate, reducing the accumulation of dental calculus in 47\%. The forms of HMP inclusion in the dry food exerted no significant effect upon calculus formation. However, there was a reduction of calculus accumulation when TPF was incorporated as coating of the kibble, compared to the inclusion of this phosphate into the interior of the kibble. It was concluded that phosphates incorporated in the dry food reduce the accumulation of dental calculus in dogs.
\end{abstract}

Keywords: dog, dental calculus, sodium tripolyphosphate, sodium hexametaphosphate

Recebido em 2 de abril de 2008

Aceito em 12 de novembro de 2008

Endereço para correspondência (corresponding adress)

Praça Santo Antônio, 44, apt.101 - 37200-000 - Lavras, MG

E-mail: adrianabrasilfp@yahoo.com.br 


\section{INTRODUÇÃO}

A doença periodontal é a afecção mais comumente encontrada na prática clínica de pequenos animais, apresentando, nos seus mais variados graus, uma prevalência de $80 \%$ a $85 \%$ nos cães adultos (Emily e Penman, 1994; Emily, 1996).

Com a evolução da medicina veterinária e, conseqüentemente, de seu segmento odontológico, maior atenção passou a ser dada à saúde bucal dos animais de estimação, uma vez que sua importância sobre o estado geral da saúde foi reconhecida. A relevância dos cuidados odontológicos sustenta-se no fator primário de comprometimento da capacidade de alimentação dos animais, o que concorre diretamente com o ganho de peso, além de predispor o animal a doenças sistêmicas graves. Há estudos mostrando que a média de vida dos animais vem aumentando desde as últimas três décadas, em parte, pelo maior cuidado com a saúde bucal (Gioso, 2001).

Com etiologia multifatorial, a doença periodontal tem como fator determinante o acúmulo de placa bacteriana sobre os dentes e tecidos adjacentes com posterior calcificação da mesma, facilitada pelo $\mathrm{pH}$ alcalino da saliva dos cães, formando, então, o cálculo dentário. $\mathrm{O}$ acúmulo de placa e cálculo leva ao quadro de gengivite $\mathrm{e}$ periodontite, caracterizando a periodontopatia ou doença periodontal (Gioso, 2001).

Vários estudos têm sido conduzidos a fim de avaliar a influência nutricional sobre a saúde bucal. Os meios dietéticos citados com maior freqüência na literatura para prevenir ou retardar o surgimento da doença periodontal são o conteúdo nutricional, a textura e a forma do alimento, os exercícios de mastigação e os alimentos ou os brinquedos tratados com produtos químicos. A estratégia padrão para evitar o acúmulo de cálculo dentário envolve o emprego de raspagem mecânica (Harvey et al., 1996; Gorrel e Rawlings, 1996a,b; Gorrel e Bierer, 1999; Gorrel et al., 1999; Hennet et al., 2004). Isso foi alcançado, basicamente, alterando-se a textura e o tamanho do grânulo da ração, porém, exerce efeito apenas nos dentes utilizados para mastigar. Uma nova abordagem utiliza a inclusão de fontes minerais nutricionais nos alimentos de maneira que possam proporcionar benefícios em todas as superfícies dentárias. Os fosfatos formam complexos solúveis com o cálcio presente na saliva, ajudando a prevenir a mineralização da placa em cálculo. Dessa forma, agem em toda cavidade bucal, inclusive nas superfícies não envolvidas na mastigação (Stookey et al., 1995; Stookey et al., 1996).

Considerando a importância de uma perfeita saúde bucal, o presente trabalho foi desenvolvido com o objetivo de avaliar o efeito de dois diferentes fosfatos, tripolifosfato de sódio e hexametafosfato de sódio, incorporados à ração seca sob duas diferentes formas, como cobertura do grânulo ou no interior da massa, na prevenção e retardo do desenvolvimento de cálculo dentário em cães.

\section{MATERIAL E MÉTODOS}

Foram utilizados 25 cães da raça Foxhound Americano, saudáveis, machos e fêmeas, não castrados, com idade média de cinco anos e peso médio de $30 \mathrm{~kg}$. Os animais foram escolhidos com base em um exame clínico criterioso, descartando-se aqueles que apresentaram afecções que pudessem interferir na condução e/ou nos resultados experimentais. Todos os cães apresentavam dentição completa, ausência de dentes decíduos, oclusão normal e ausência de afecções periodontais prévias. Os animais foram distribuídos de forma aleatória em cinco tratamentos com cinco repetições. Os tratamentos experimentais foram: 1) ração seca; 2) ração seca com tripolifosfato de sódio (TPF) incorporado na cobertura do grânulo da ração; 3 ) ração seca com TPF incorporado no interior da massa da ração; 4) ração seca com hexametafosfato de sódio (HMF) incorporado na cobertura do grânulo da ração e 5) ração seca com HMF incorporado no interior da massa da ração. Em todas as dietas experimentais, a concentração do fosfato foi de $300 \mathrm{ppm}$. A quantidade de ração fornecida foi calculada de acordo com as necessidades energéticas de cada animal, determinadas por meio da equação de predição de Heusner (1982), citada pelo NCR (Nutrient..., 1985), acrescida de 10\%. Os tratamentos foram oferecidos uma vez ao dia, por volta das $12 \mathrm{~h} 30 \mathrm{~min}$, por tratadores experientes. Todos os cães apresentaram consumo adequado do alimento oferecido. Além das dietas 
experimentais, os cães receberam água fresca à vontade.

Os animais receberam as dietas por um período de adaptação de sete dias e, em seguida, foram submetidos à profilaxia dentária - extração de cálculo e polimento dentários. Após um período experimental de 90 dias, os animais foram submetidos à avaliação da área de cálculo dentário formado sobre a face vestibular dos seguintes dentes unilaterais (hemiarcada direita): canino (C), segundo pré-molar (P2), terceiro prémolar (P3), quarto pré-molar (P4), primeiro molar (M1), maxilares e mandibulares. A mensuração do cálculo dentário foi feita de acordo com índices clínicos modificados de Greene e Vermillion (1960) e Volpe e Manhold (1965). A avaliação da superfície do dente coberta pelo cálculo dentário seguiu os índices: 0 $=$ nenhum cálculo observável; $1=$ cálculo cobrindo menos de $10 \%$ da superfície; $2=$ entre $10 \%$ e $33 \%$ de cobertura; $3=$ entre $33 \%$ e $66 \%$ de cobertura; $4=$ mais de $66 \%$ de cobertura. A espessura do depósito de cálculo em cada dente foi avaliada de acordo com os seguintes índices: $1=$ menos que $0,5 \mathrm{~mm}$ de espessura, leve; $2=\mathrm{de}$ 0,5 a $1,0 \mathrm{~mm}$ de espessura, moderado; $3=$ mais que $1,0 \mathrm{~mm}$ de espessura, acentuado. A área dental coberta pelo cálculo foi obtida pela multiplicação do índice de superfície pelo índice de espessura referente a cada dente estudado. A contagem numérica para cada tratamento foi obtida pela somatória dos índices de área do cálculo dentário de cada dente das repetições e o total dividido pelo número de dentes, obtendo-se, para cada animal de cada tratamento, um único valor. Todas as avaliações foram realizadas por três avaliadores em conjunto, os quais não possuíam conhecimento dos grupos de cães e seus respectivos tratamentos e não estavam diretamente envolvidos na condução do estudo.
Os dados foram submetidos à análise de variância utilizando-se o pacote computacional Sisvar for Windows. A verificação de diferença significativa entre contrastes que envolvem grupos de médias foi realizada pelo teste de Scheffé.

Os contrastes entre os tratamentos foram estruturados como se segue: ausência de fosfato $v s$ tripolifosfato de sódio em cobertura; ausência de fosfato $v s$ tripolifosfato de sódio em massa; ausência de fosfato $v s$ hexametafosfato de sódio em cobertura; ausência de fosfato vs hexametafosfato de sódio em massa; hexametafosfato de sódio $v s$ tripolifosfato de sódio; tripolifosfato em cobertura vs tripolifosfato em massa; hexametafosfato em cobertura $v s$ hexametafosfato em massa.

\section{RESULTADOS E DISCUSSÃO}

Os resultados da área do cálculo formado sobre os dentes dos cães, em cada tratamento, estão descritos na Tab. 1. Na Tab. 2, apresenta-se a análise de variância para área de cálculo formado em função dos contrastes estudados.

Tabela 1. Valores médios de área de cálculo formado em função dos tratamentos

\begin{tabular}{lc}
\hline Tratamento & $\begin{array}{c}\text { Área de cálculo } \\
\text { formado }\end{array}$ \\
\hline $\begin{array}{l}\text { Ausência de fosfato } \\
\text { Tripolifosfato em } \\
\text { cobertura }\end{array}$ & 7,78 \\
$\begin{array}{l}\text { Tripolifosfato em massa } \\
\text { Hexametafosfato em }\end{array}$ & 5,90 \\
$\begin{array}{l}\text { cobertura } \\
\text { Hexametafosfato em }\end{array}$ & 4,62 \\
massa & 5,12 \\
\hline
\end{tabular}

Tabela 2. Análise de variância para área de cálculo formado em função dos contrastes

\begin{tabular}{lccc}
\hline Fonte de variação & GL & \multicolumn{2}{c}{ Quadrado médio (P) } \\
\hline Ausência $v s$ tripolifosfato em cobertura & 1 & 8,836 & $(0,1029)$ \\
Ausência $v s$ tripolifosfato em massa & 1 & 0,064 & $(0,8858)$ \\
Ausência $v s$ hexametafosfato em cobertura & 1 & 34,225 & $(0,0031)$ \\
Ausência $v s$ hexametafosfato em massa & 1 & 17,689 & $(0,0253)$ \\
Tripolifosfato $v s$ hexametafosfato & 1 & 23,328 & $(0,0116)$ \\
Cobertura $v s$ massa (tripolifosfato) & 1 & 7,396 & $(0,1336)$ \\
Cobertura $v s$ massa (hexametafosfato) & 1 & 2,704 & $(0,3557)$ \\
Erro & 20 & \multicolumn{2}{c}{3,024600} \\
\hline Coeficiente de variação $(\%)$ & & \multicolumn{2}{c}{28,51} \\
\hline
\end{tabular}


A inclusão do hexametafosfato de sódio na ração seca, tanto na cobertura dos grânulos $(\mathrm{P}=0,0031)$ como no interior da massa $(\mathrm{P}=0,0253)$, reduziu o acúmulo de cálculo dentário em cães, quando comparada à dieta sem adição de fosfatos.

O tripolifosfato de sódio incorporado como cobertura da ração seca foi efetivo $(\mathrm{P}=0,1029)$ se comparado ao alimento sem adição de fosfatos. O tripolifosfato de sódio incorporado no interior da massa da ração seca não influenciou $(\mathrm{P}=0,8858) \quad$ o desenvolvimento de cálculo dentário se comparado ao regime sem adição de agente quelante.

Os resultados indicam a habilidade dos fosfatos em diminuir a formação de cálculo dentário em cães, quando adicionados às rações secas comerciais. Stookey et al. (1995) constataram a efetividade de dois tipos de fosfatos, hexametafosfato de sódio e pirofosfato solúvel, incorporados na ração seca como cobertura, sobre a redução do depósito de cálculo dentário em $76,8 \%$ e $47,2 \%$, respectivamente. Três experimentos, no estudo de Stookey et al. (1995), mostraram que biscoitos cobertos com hexametafosfato de sódio diminuíram o acúmulo de cálculo dentário em $62,8 \%$ a $79,6 \%$, numericamente superiores aos valores encontrados no presente estudo - $24 \%$ a $47 \%$ (Tab. 3). Provavelmente, tal superioridade devese a dois fatores: a utilização de ração semiúmida como dieta basal, que facilita maior acúmulo de cálculo sobre os dentes, comparada às rações secas, e a adição dos fosfatos em biscoitos, os quais promovem um exercício de mastigação maior que a ração seca convencional. Stookey et al. (1996), ao utilizarem ração seca como dieta base e fornecerem diariamente dois biscoitos cobertos com hexametafosfato de sódio, verificaram redução de $46 \%$ sobre a formação do cálculo dentário. Paiva et al. (2007) observaram redução do acúmulo de placa dentária em cães tratados com biscoitos enriquecidos com tripolifosfato de sódio ou hexametafosfato de sódio, comparados ao regime com biscoitos convencionais sem a adição de fosfatos.

O hexametafosfato de sódio foi o fosfato mais eficiente $(\mathrm{P}=0,0116)$, comparado ao tripolifosfato de sódio, pois reduziu o acúmulo de cálculo dentário em $34,2 \%$ e 47,6\%, em massa e em cobertura, respectivamente (Tab. 3).
Tabela 3. Porcentagem de redução do acúmulo de cálculo dentário em cães de acordo com os tratamentos

\begin{tabular}{lcc}
\hline Tratamento & $\begin{array}{c}\text { Área de } \\
\text { cálculo }\end{array}$ & Redução \\
\hline Ausência de fosfato & 7,78 & \\
TFP em cobertura & 5,90 & $24,2 \%$ \\
HMF em massa & 5,12 & $34,2 \%$ \\
HMF em cobertura & 4,08 & $47,6 \%$ \\
\hline
\end{tabular}

TFP: tripolifosfato de sódio; HMF: hexametafosfato de sódio.

Estes resultados diferem de resultados prévios. Hennet et al. (2005) compararam o efeito do tripolifosfato de sódio e do hexametafosfato de sódio adicionados como cobertura da ração seca em 20 cães da raça Beagle, por um período de quatro semanas. $\mathrm{O}$ depósito de cálculo sobre os dentes foi reduzido em $55 \%$ para o tripolifosfato de sódio e em 36\% para o hexametafosfato de sódio, quando comparada à redução proporcionada pelo grupo sem a adição de fosfatos; entretanto, não houve diferença significativa entre os fosfatos estudados.

Paiva et al. (2007) também não encontraram diferenças significativas na redução do acúmulo de placa bacteriana entre os cães tratados, por um período de 21 dias, com biscoitos contendo tripolifosfato de sódio e os tratados com biscoitos contendo hexametafosfato de sódio. A contradição entre os resultados encontrados por esses autores e os do presente estudo pode ser atribuída a diferentes metodologias, incluindo a fonte de fornecimento do fosfato, ração seca $v s$ biscoitos, e o tempo de fornecimento dos tratamentos experimentais, 90 dias $v s 30$ dias ou 21 dias.

Stookey et al. (1995) verificaram que o hexametafosfato de sódio foi mais eficiente em reduzir o acúmulo de cálculo dentário, tanto na cobertura da ração seca $(29,6 \%$ mais eficaz), como na cobertura de biscoitos $(32,7 \%$ mais eficaz), que o pirofosfato solúvel. A maior eficiência do hexametafosfato de sódio em impedir a mineralização da placa dentária, complexando o cálcio presente no fluido salivar, pode estar relacionada à sua estrutura química. De acordo com White e Gerlach (2000), diferentes estruturas conferem diferentes propriedades químicas aos agentes quelantes. Acredita-se que o maior tamanho do complexo iônico do hexametafosfato de sódio, comparado 
aos complexos iônicos do tripolifosfato de sódio e do pirofosfato de sódio, seja responsável por sua maior eficácia.

A forma de inclusão do hexametafosfato de sódio na ração, como cobertura do grânulo ou no interior da massa, não se constituiu em fonte de variação na redução do acúmulo de cálculo dentário. Os fosfatos são liberados durante a mastigação e difundem-se pela saliva, agindo em toda a cavidade bucal, inclusive nas superfícies não envolvidas na mastigação, assim, a forma de inclusão não influenciaria o mecanismo de ação do fosfato.

Cox e Lepine (2002) verificaram redução de $58 \%$ sobre a formação de cálculo dentário quando o hexametafosfato de sódio foi usado na cobertura do grânulo, se comparada à sua inclusão no interior da massa da ração. Stookey et al. (1995) também observaram diferenças significativas entre as formas de inclusão dos fosfatos nos alimentos; o efeito anticálculo atribuído ao pirofosfato solúvel foi significativo somente quando usado na camada superficial dos grânulos da ração ou dos biscoitos. Uma razão que poderia explicar esses resultados seria que, contrariamente aos seres humanos, os cães apresentam atividade mastigatória reduzida, deglutindo os alimentos com grande voracidade, sem quebrá-los completamente, dificultando, portanto, a liberação dos fosfatos presentes no interior do grânulo da ração.

\section{CONCLUSÕES}

Os fosfatos empregados foram efetivos em reduzir o depósito de cálculo sobre os dentes dos cães. A inclusão do fosfato na ração seca é uma opção prática para o controle do cálculo dentário. $\mathrm{O}$ hexametafosfato de sódio foi superior ao tripolifosfato de sódio na redução do acúmulo de cálculo dentário, e pode ser usado tanto como cobertura dos grânulos quanto na massa da ração sem comprometimento de sua eficácia. O tripolifosfato de sódio mostrou-se efetivo somente quando usado na camada superficial dos grânulos da ração.

\section{REFERÊNCIAS BIBLIOGRÁFICAS}

COX, E.R.; LEPINE, A.J. Use of polyphosphate in canine diets to control tartar. In: GENERAL SESSION \& EXHIBITION OF THE IADR, 80.,
2002, San Diego. Proceedings... San Diego, 2002. (A349).

EMILY, P. Diagnóstico e profilaxia de uma periodontopatia. In: BOJRAB, M.J. (Eds). Técnicas atuais em cirurgia de pequenos animais. 3.ed. São Paulo: Roca, 1996. p.158-162.

EMILY, P.; PENMAN, S. Handbook of small animal dentistry. 2.ed. Oxford: Pergamon, 1994. $117 \mathrm{p}$.

GIOSO, M.A. Odontologia veterinária para o clínico de pequenos animais. 4.ed. São Paulo: FMVZ-USP, 2001.

GORREL, C.; BIERER, T.L. Long-term effects of a dental hygiene chew on the periodontal health of dogs. J. Vet. Dent., v.16, p.109-113, 1999.

GORREL, C.; RAWLINGS, J.M. The role of a 'Dental Hygiene Chew' in maintaining periodontal health in dogs. J. Vet. Dent., v.13, p.31-34, 1996a.

GORREL, C.; RAWLINGS, J.M. The role of tooth-brushing and diet in maintaining periodontal health in dogs. J. Vet. Dent., v.13, p.139-143, 1996b.

GORREL, C.; WARRICK, J.; BIERER, T.L. Effect of a new dental hygiene chew on periodontal health in dogs. J. Vet. Dent., v.16, p.77-81, 1999.

GREENE, J.C.; VERMILLION, J.R. The oral hygiene index: a method for classifying oral hygiene status. J. Am. Dent. Assoc., v.61, p.172$179,1960$.

HARVEY, C.E.; SHOFER, F.S.; LASTER, L. Correlation of diet, other chewing activities and periodontal disease in North American clientowned dogs. J. Vet. Dent., v.13, p.101-105, 1996.

HENNET, P.; SERVET, E.; SOULARD, Y. et al. Effects of a two kibbles size and two different phosphate salts in preventing calculus accumulation in dogs. In: EUROPEAN CONGRESS OF VETERINARY DENTISTRY, 14., 2005, Ljubljana. Proceedings... Ljubljana, 2005.

HENNET, P.; SERVET, E.; VENET, C. Effects of feeding a daily oral hygiene chew on dental deposits in small breed dogs: a 4-month trial. In: EUROPEAN CONGRESS OF VETERINARY 
DENTISTRY, 13., 2004, Krakov. Proceedings... Krakow, 2004. p.47-48.

HEUSNER, A.A. Energy metabolism and body size I: is the 0.75 mass exponent of Kleiber's equation a statistical artifact? Respir. Physiol., v.48, p.1-12, 1982.

NUTRIENT requirements of dogs. Washington: National Academy, 1985. 81p.

PAIVA, A.C.; SAAD, F.M.O.B.; LEITE, C.A.L. et al. Eficácia dos coadjuvantes de higiene bucal utilizados na alimentação de cães. Arq. Bras. Med. Vet. Zootec., v.59, p.1177-1183, 2007.

STOOKEY G.K.; WARRICK J.M.; MILLER L.L. Effect of sodium hexametaphosphate on dental calculus formation in dogs. Am. J. Vet. Res., v.56, p.913-918, 1995.

STOOKEY, G.K.; WARRIK, J.M.; MILLER, L.L. et al. Hexametaphosphate-coated snacks biscuits significantly reduce calculus formation in dogs. J. Vet. Dent., v.13, p.27-30, 1996.

VOLPE, A.R.; MANHOLD, J.H.; HAZEN, S.P. In vivo calculus assessment: Part I. A method and its examiner reproducibility. J. Periodontol., v.36, p.292-298, 1965.

WHITE, D.J.; GERLACH, R.W. Anticalculus effects of a novel, dual-phase polypyrophosphate dentifrice: chemical basis, mechanism, and clinical response. J. Contemp. Dent. Pract., v.1, p.1-19, 2000. 